\title{
A Numerical Method for \\ Optimal Control-State Problem with Bivariate B-Spline Basis
}

\author{
Ali Zakeri ${ }^{a, b}$, Mohammad Masjed-Jamei ${ }^{b}$, Amir Hossein Salehi Shayegan $^{b}$ \\ ${ }^{a}$ Department of Mathematics, South Tehran Branch, Islamic Azad University, Tehran, Iran, \\ azakeri@kntu.ac.ir \\ ${ }^{b}$ Department of Mathematics, K. N. Toosi University of Technology, P. O. Box 16315-1618, Tehran, \\ Iran, \\ mmjamei@kntu.ac.ir,mmjamei@yahoo.com,asalehi@sina.kntu.ac.ir
}

Article history:

Received March 2013

Accepted May 2013

Available online July 2013

\begin{abstract}
We apply the bivariate B-spline basis to find an approximate solution for control-state function in a constrained optimal control problem, whose constraint is an elliptic partial differential equation (PDE) with Dirichlet boundary conditions. In this method, the PDE is first discretized and then by using bivariate B-spline basis, a state function is obtained with respect to some unknown coefficients. By applying generalized Newton method, the optimal value for the control function is also determined. Finally, a numerical example is given and the optimal solution is derived by using the bivariate B-spline basis.
\end{abstract}

Keywords: Bivariate B-spline basis, Optimal control problem, Elliptic equation, WEBspline finite element method.

\section{Introduction}

The optimization of a control-state problem was first proposed by Hinze [6]. He considered an optimization problem subject to some constrains in terms of PDE with additional constrains on the control-state $[4,6]$. This problem is today one of the most important problems in industrials and economical applications. In general, since it cannot be solved by analytic methods, numerical methods should be applied in this sense.

Before 1980s, indirect methods were used for solving optimal control problems. To obtain the first order optimality conditions in these methods, we usually use the calculus of variations, see e.g. [6,9].

After that and for two decades up to now direct methods are used [6]. In a direct method, the state and control functions are approximated using an appropriate numerical method. 
Simultaneously, the cost functional is approximated as a discrete function. Hence, the coefficients of the approximate function are treated as optimization variables and the problem is transformed to a nonlinear optimization problem. Depending upon the type of the employed direct method, nonlinear optimal control problems can be quite small or large $[6,9]$.

Hinze in [6] and Zakeri and Asadi in [4] applied the finite and boundary element methods for solving this kind of problems, respectively. In this paper, we apply bivariate Bspline basis in finite element methods, which is actually efficient for reducing order of the system. This method first introduced by Klaus Hollig in [7] and then recalled as WEBspline finite element method. Some advantages of the mentioned method is given in third section.

In this paper, we consider an optimal control problem with PDE constraints proposed by Hinze and obtain an approximate solution for the state and control function by using WEB-spline finite element method. For this purpose in section 2, by using the results of [6,9], the mathematical formulation of an optimal control problem with PDE constraints is provided. In section 3, the finite element method with bivariate B-spline basis, is explained and finally in the last section, we consider an example provided by Hinze in [6] to show the accuracy and efficiency of the propose method.

\section{Mathematical formulation}

Let $\alpha>0$ be a known constant and $Y=H_{0}^{1}(\Omega)$ where $\Omega \subset \mathbb{R}^{2}$ is the computation domain. Also let $H$ and $U$ denote Hilbert spaces, $R$ and $Z$ Banach spaces and $Y_{a d}$ and $U_{a d}$ be respectively representations of convex and closed subspaces $Y$ and $U$. For a known function $y_{0}$, let $y$ and $u$ be unknown state and control functions. By finding them, the functional

$$
J(y, u):=\frac{1}{2}\left\|y-y_{0}\right\|_{L^{2}(\Omega)}^{2}+\frac{\alpha}{2}\|u\|_{U}^{2}, \quad y, u \in Y \times U
$$

with the constraints

$$
\left\{\begin{aligned}
-\Delta y=u & \text { in } \Omega \in U_{a d} \subset U \\
y=0 & \text { on } \partial \Omega
\end{aligned}\right.
$$

should be minimized in the computational domain $\Omega$. This problem can be transformed to a linear-quadratic optimization problem of the form

$$
\begin{aligned}
& \min _{(y, u) \in Y \times U} J(y, u):=\frac{1}{2}\left\|Q y-q_{d}\right\|_{H}^{2}+\frac{\alpha}{2}\|u\|_{U}^{2} \\
& \text { subject to } \quad A y+u=g, \quad u \in U_{a d}, y \in Y_{a d}
\end{aligned}
$$

where $q_{d} \in H, g \in Z, A \in L \quad Y, Z$ and $Q \in Y, H$. In what follows, we express existence results for the general linear-quadratic problem (3) according to [6].

Theorem 2.1. ([4, 6]) Suppose that the following assumptions hold:

- $\quad U_{a d} \subset U$ is convex and closed for $\alpha>0$ and bounded for $\alpha=0$.

- $Y_{a d} \subset Y$ is a convex and closed, such that (3) has a feasible point.

- $A \subset L(Y, Z)$ has a bounded inverse.

Then the problem (3) has an optimal solution $\bar{y}, \bar{u}$. Also if $\alpha>0$, then this solution is unique. 
Proof. The proof with details are given in [6].

Remark 2.2. A state-control pair $\bar{y}, \bar{u} \in Y_{a d} \times U_{a d}$ is called optimal in (3), if $A \bar{y}+\bar{u}=g$ and

$$
J \bar{y}, \bar{u} \leq J \quad y, u, \quad \forall y, u \in Y_{a d} \times U_{a d}, A y+u=g .
$$

In the next section, by referring to the results [2, 3, 7 and 8], we introduce the bivariate Bspline basis and apply it in finite element method as basis functions.

\section{Bivariate B-spline basis}

Following the notations in [7], our aim in this section is to apply bivariate B-spline basis in the finite element method as the basis functions. B-spline tensor product is an extension of $\mathrm{B}$-spline in higher dimensions. So, in order to make a bivariate B-spline of degree $n, b_{\mathbf{k}, h}^{n}$, ,one dimensional B-spline tensor product is used as follows

$$
b_{\mathbf{k}, h}^{n}(x, y)=b_{k_{1}, h}^{n}(x) \otimes b_{k_{2}, h}^{n}(y), \quad \mathbf{k}=\left(k_{1}, k_{2}\right) \in K
$$

in which $b_{\ell, h}^{n}(x)=b^{n}\left(\frac{x}{h-\ell}\right)$,

and

$$
b^{n}(x)=\frac{x}{n} b^{n-1}(x)+\frac{n+1-x}{n} b^{n-1}(x-1), \quad(n=2,3, \ldots)
$$

$$
b^{1}(x)=\left\{\begin{array}{lc}
x & 0 \leq x \leq 1 \\
2-x & 1 \leq x \leq 2 \\
0 & \text { otherwise }
\end{array}\right.
$$

In the above formula, the set $K$ includes all $\mathbf{k}$ indices in such a way that for some $\mathbf{x} \in \Omega$, $b_{\mathbf{k}, h}^{n} \neq 0$ and $h$ is increment of variables $x$ and $y$.In addition, assume that the support of bivariate B-spline basis is determined by $k_{1}, k_{1}+n+1 h \times k_{2}, k_{2}+n+1 h$.

Now, $b_{\mathbf{k}, h}^{n} \quad \mathbf{k} \in K$ are divided into two sets, i.e. inner B-splines and outer B-splines. $b_{\mathbf{k}, h}^{n}$ is an inner B-spline for $\mathbf{k} \in K$ only when the $\operatorname{support} b_{\mathbf{k}, h}^{n}$ has at least one grid cell which is completely located in $\Omega$.

Otherwise every B-spline is considered an outer B-spline. In other words, the set of $K$ indices split into two subsets, indices of inner B-splines, $I$, and indices of outer B-splines, $J$.

Applying the above bivariate B-spline basis in a finite element method as basis functions may seem impossible, because these basis do not follow the essential boundary conditions. To resolve this problem, we can use the smoothed distance function $\omega(\mathbf{x})=\operatorname{dist}(\mathbf{x}, \partial \Omega) \quad(\mathbf{x} \in \Omega)$, which is thoroughly defined as a continuous function and is positive on $\omega$ and negative on complement $\bar{\Omega}$, and then multiply $b_{\mathbf{k}, h}^{n}$ by this function, e.g. refer to the Rvachev method (or R-function), which is used in numerical techniques [7].

We can construct a weighted B-spline space by multiplying $\omega(\mathbf{x})$ and $b_{\mathbf{k}, h}^{n}$ respectively and consider it as a subspace of finite elements for the Dirichlet boundary value problems, which would give us better approximations. However, since outer B-splines have a small support in $\Omega$, the condition number of Galerkin matrix might be extremely large. A suitable solution for the problem of controlling the unstable outer B-splines is provided by appropriately adjoining them with the inner B-splines. Using the above mentioned process, we can have access to the weighted B-spline space again and call it weighted extended B- 
spline space $\omega^{e} B_{h}^{n}(\Omega)$. Therefore, for every $\mathbf{i} \in I$, we define weighted extended B-spline $B_{\mathbf{i}}$ (WEB-spline) as follows

$$
B_{\mathbf{i}}=\frac{\omega}{\omega\left(\mathbf{x}_{\mathbf{i}}\right)}\left(b_{\mathbf{i}}+\sum_{\mathbf{j} \in J} e_{\mathbf{i j}} b_{\mathbf{j}}\right), \quad\left(b_{\mathbf{k}}=b_{\mathbf{k}, h}^{n}\right),
$$

in which $\mathbf{x}_{\mathbf{i}}$ show the center of a grid cell in the relation support $b_{\mathbf{i}}$ which is completely located in $\Omega$. Also the coefficients $e_{\mathbf{i j}}$ satisfy $e_{\mathbf{i j}}=0$ for $\|\mathbf{i}-\mathbf{j}\| \geq 1$ and $\left|e_{\mathbf{i j}}\right| \leq 1$. To calculate $e_{\mathrm{ij}}$ the starting point is the B-spline representation of polynomials. Marsden in [7] proved that every polynomial $p$ of coordinate degree $n$ can be written as a linear combination in the form

$$
p(\mathbf{x})=\sum_{\mathbf{i} \in I} q(\mathbf{i}) b_{\mathbf{i}}(\mathbf{x})+\sum_{\mathbf{j} \in J} q(\mathbf{j}) b_{\mathbf{j}}(\mathbf{x}) \quad(\mathbf{x} \in \Omega)
$$

Our goal is to associate the inner B-spline with outer B-spline using $e_{\mathrm{ij}}$ coefficients. For outer index $\mathbf{j} \in J$ suppose that $I(\mathbf{j})=\ell+\{0, \ldots, n\}^{2} \subset I$, is a two dimensional array of inner indices close to $\mathbf{j}$. Since $q$ is a polynomial of coordinate degree $n$, coefficients $q(\mathbf{j}), \mathbf{j} \in J$ can be calculated using $\mathbf{i} \in I(\mathbf{j}) \subset I$ indices. Now, we consider $q$ as a polynomial interplant with the values $q(\mathbf{i}), \mathbf{i} \in I(\mathbf{j})$ and define Lagrange polynomials of degree $n$ in accordance to $I(\mathbf{j})$ and $L_{\mathrm{ji}}$. In other words, we have

$$
L_{\mathbf{j i}}(\mathbf{k})=\delta_{\mathbf{i k}}, \quad \mathbf{i}, \mathbf{k} \in I(\mathbf{j}),
$$

then for fixed $\mathbf{j}, e_{\mathbf{i j}}$ are placed in the point of $\mathbf{j}$ as the value of Lagrange polynomials. Therefore,

$$
e_{\mathbf{i j}}=L_{\mathbf{j i}}(\mathbf{j}), \quad \mathbf{i} \in I(\mathbf{j}) .
$$

According to [7], for simplification, we put $e_{\mathrm{ij}}=0$ for each $\mathbf{i} \in I(\mathbf{j})$. Hence

$$
q(\mathbf{j})=\sum_{\mathbf{i} \in I(\mathbf{j})} e_{\mathbf{i j}} q(\mathbf{i}) .
$$

If we replace the latter relation in (4), we get

$$
p(\mathbf{x})=\sum_{\mathbf{i} \in I} q(\mathbf{i})\left(b_{\mathbf{i}}(\mathbf{x})+\sum_{\mathbf{j} \in J} e_{\mathbf{i j}} b_{\mathbf{j}}(\mathbf{x})\right) .
$$

This completes the process of making $e_{\mathrm{ij}}$. Analyzing the above said process leads to the following definition, mentioned in [7].

Definition 3.1. For an outer index $\mathbf{j} \in J$ let $I(\mathbf{j})=\ell+\{0, \ldots, n\}^{2} \subset I$ be an two-dimensional array of inner indices close to $\mathbf{j}$, assuming that $h$ is small enough so that such an array exists. Moreover, denote by

$$
e_{\mathrm{ij}}=\prod_{v=1}^{2} \prod_{u=0, \ell_{v}+\mu \neq i_{v}}^{n} \frac{j_{v}-\ell_{v}-\mu}{i_{v}-\ell_{v}-\mu}
$$

the values of the Lagrange polynomials associated with $I(\mathbf{j})$.

According to $[2,7]$, we give some features of definition $B_{\mathbf{i}}$ as follows 
- Because of the linear independence of B-splines, WEB-splines are linearly independent, too.

- The factor $\omega / \omega\left(\mathbf{x}_{\mathbf{i}}\right)$ causes the WEB-splines to vanish on the boundary and magnifies functions supported near the boundary for scaling purpose. This fact will become important for proving the stability aspect of the WEB-splines.

- By forming linear combinations, the support of a WEB-spline is in general bigger than that of a B-spline. However, restricting nonzero coefficient $e_{\mathrm{ij}}$ to indices with $\|\mathbf{i}-\mathbf{j}\| \leq 1$ guarantees that the diameter of the support of WEB-spline is still $\leq h$. In particular, WEB-splines with support sufficiently wellseparated from the boundary are just ordinary B-spline multiplied by $\omega / \omega\left(\mathbf{x}_{\mathbf{i}}\right)$. Hence, only $\leq h^{1-m}$ WEB-splines involve linear combinations of outer B-splines.

- The uniform boundedness of the coefficients $e_{\mathrm{ij}}$, prevents the WEB-spline from growing in an uncontrolled way as the grid width $h$ is tending to zero.

Now, let

$$
y(\mathbf{x})=\sum_{\mathbf{i} \in I} c_{\mathbf{i}} B_{\mathbf{i}}(\mathbf{x}), \quad(\mathbf{x} \in \Omega)
$$

where $c_{\mathbf{i}},(\mathbf{i} \in I)$ are unknown and $B_{\mathbf{i}}$ are WEB-splines defined above. To obtain $c_{\mathbf{i}}$, $(\mathbf{i} \in I)$, we encounter with the linear system of equations derived by the Ritz-Galerkin approximation of Poisson's problem (2). In fact we should solve the a nonsingular linear system of equations as

$$
\mathbf{A C}=\mathbf{F}
$$

where

$$
\mathbf{A}=\left\{A_{i j}\right\}_{K \times K} \quad \text { with } \quad A_{i j}=\int_{\Omega} \nabla B_{\mathbf{i}} \cdot \nabla B_{\mathbf{j}} d \mathbf{x} \text { and } \quad \mathbf{C}=\left(c_{1}, c_{2}, \ldots, c_{K}\right)^{T},
$$

and

$$
\mathbf{F}=\left(f_{1}, f_{2}, \ldots, f_{K}\right)^{T} \quad \text { with } \quad f_{i}=\int_{\Omega}(u) B_{\mathbf{i}} d \mathbf{x},
$$

in which $K$ is the number of inner B-splines. Note that $\operatorname{Cond}(\mathbf{A}) \leq h^{-2}$ ( see [3]).

By solving (6) and substituting the coefficients $c_{\mathbf{i}}$ in (5) an approximate solution for Poisson's problem (2) can be derived.

In the sequel by using the results of [1, 2, 3, 7 and 8], we study some advantages of WEBspline finite element method and then provide a numerical algorithm for the control-state problem (1-2).

Some advantages of WEB-spline finite element method are respectively, no mesh generation is required, the uniform grid is ideally suited for parallelization and multigrid techniques, accurate approximations are possible with relatively low-dimensional subspaces, smoothness and approximation order can be chosen arbitrarily and finally hierarchical bases permit adaptive refinement.

\section{A numerical algorithm for the given control-state problem}

- Let $u\left(x_{1}, x_{2}\right)=s_{1}+s_{2} x_{1}+s_{3} x_{2}+s_{4} x_{1} x_{2} \in L^{2}(\Omega)$, where $s_{1}, s_{2}, s_{3}$ and $s_{4} \quad$ are the unknown coefficients.

- Solve Poisson's problem: 


$$
\left\{\begin{array}{cl}
-\Delta y=s_{1}+s_{2} x_{1}+s_{3} x_{2}+s_{4} x_{1} x_{2}, & \text { in } \Omega \\
y=0 & \text { on } \Omega,
\end{array}\right.
$$

using the WEB-spline finite element method and obtain an approximate solution for the state function $y$, ( show it by $\bar{y})$. Clearly, we should first solve the following problems

$$
\begin{aligned}
& \left\{\begin{aligned}
-\Delta y_{1}=1 & \text { in } \Omega \\
y_{1}=0 & \text { on } \partial \Omega,
\end{aligned}\right. \\
& \left\{\begin{array}{rll}
-\Delta y_{2}=x_{1} & \text { in } & \Omega \\
y_{2}=0 & \text { on } & \partial \Omega,
\end{array}\right. \\
& \left\{\begin{array}{rrl}
-\Delta y_{3}=x_{2} & \text { in } & \Omega \\
y_{3}=0 & \text { on } & \partial \Omega,
\end{array}\right.
\end{aligned}
$$

and

$$
\left\{\begin{array}{ccc}
-\Delta y_{4}=x_{1} x_{2} & \text { in } & \Omega \\
y_{4}=0 & \text { on } & \partial \Omega
\end{array}\right.
$$

and then take $\bar{y}=s_{1} \bar{y}_{1}+s_{2} \bar{y}_{2}+s_{3} \bar{y}_{3}+s_{4} \bar{y}_{4}$, where $\bar{y}_{1}, \bar{y}_{2}, \bar{y}_{3}$ and $\bar{y}_{4}$ are the approximate solutions of the above problems.

- Replace the state and control function $\bar{y}$ and $u\left(x_{1}, x_{2}\right)=s_{1}+s_{2} x_{1}+s_{3} x_{2}+s_{4} x_{1} x_{2}$ in (1).

- Apply the generalized Newton method and compute unknown values $s_{1}, s_{2}, s_{3}$ and $s_{4}$.

\section{A Numerical Example}

In this section, to show the accuracy and efficiency of the WEB-spline finite element method for the optimal control-state problem (1-2), we consider the following problem, which is expressed by Hinze in [6]

$$
\underset{(y, u) \in L^{2}(\Omega) \times L^{2}(\Omega)}{\operatorname{Min}} J(y, u):=\frac{1}{2}\left\|y+\operatorname{sign}\left(x_{1}-0.5-\frac{0.1}{\pi}\right)\right\|_{L^{2}(\Omega)}^{2}+\frac{1}{2}\|u\|_{L^{2}(\Omega)}^{2},
$$

subject to

$$
\left\{\begin{aligned}
-\Delta y=u, & \text { in } \quad \Omega=[0,1] \times[0,1-x], \quad u \in U_{a d} \subseteq L^{2}(\Omega) \\
y=0 & \text { on } \quad \partial \Omega
\end{aligned}\right.
$$

where $y$ and $u$ are the state and control functions, respectively.

Let $u\left(x_{1}, x_{2}\right)=s_{1}+s_{2} x_{1}+s_{3} x_{2}+s_{4} x_{1} x_{2}$ where $s_{1}, s_{2}, s_{3}$ and $s_{4}$ are unknown constant coefficients. We solve the Poisson equation with Dirichlet boundary conditions using WEB-spline finite element method in which the step size is considered $h=0.5,0.25$ and the weight function (Rvachev method) is as

$$
\omega\left(x_{1}, x_{2}\right)=1-\sqrt{x_{1}^{2}+x_{2}^{2}}-\sqrt{\left(1-x_{1}-x_{2}\right)^{2}+\left(x_{1}+x_{2}-\sqrt{x_{1}^{2}+x_{2}^{2}}\right)^{2}} .
$$

Then we determine the state function $y$ depending on the unknown parameters $s_{1}, s_{2}, s_{3}$ and $s_{4}$.

By substituting $y$ and $u\left(x_{1}, x_{2}\right)=s_{1}+s_{2} x_{1}+s_{3} x_{2}+s_{4} x_{1} x_{2}$ in the functional $J$, we reach a nonlinear function with respect to pour variables $s_{1}, s_{2}, s_{3}$ and $s_{4}$. 
In order to minimize the functional $J$, the generalized Newton method can be used and the optimal value of $J$ is therefore obtained. In this example, by using 20 iterations of the Newton method, we have obtained the unknown values $s_{1}, s_{2}, s_{3}, s_{4}$ and the optimal value $J$. Numerical results are listed in tables $1,2,3$ and the function $u\left(x_{1}, x_{2}\right)$ is shown in figures 1 and 2.

\begin{tabular}{|c|c|c|c|c|c|}
\hline$h$ & $s_{1}$ & $s_{2}$ & $s_{3}$ & $s_{4}$ & $J$ \\
\hline \hline 0.5 & -0.0204769 & 0.0318994 & 0.0188897 & -0.0742583 & 0.25003 \\
\hline 0.25 & 0.0195079 & -0.0296133 & -0.0175944 & 0.0637552 & 0.249974 \\
\hline
\end{tabular}

Table 1. Control function $u$ for different iterations by the iterative Newton method.

\begin{tabular}{|c|c|c|c|c|}
\hline$\left(x_{1}, x_{2}\right)$ & $x_{1}=0.2$ & $x_{2}=0.4$ & $x_{3}=0.6$ & $x_{4}=0.8$ \\
\hline \hline$x_{1}=0.2$ & -0.0132895 & -0.00987991 & -0.00647036 & -0.00306081 \\
\hline$x_{2}=0.4$ & -0.0124819 & -0.0120426 & -0.0116034 & - \\
\hline$x_{3}=0.6$ & -0.0116743 & -0.0142054 & - & - \\
\hline$x_{4}=0.8$ & -0.0108667 & - & - & - \\
\hline
\end{tabular}

Table 2. Function $u\left(x_{1}, x_{2}\right)$ with 20 iterations by the Newton method and the step size $h=0.5$.

\begin{tabular}{|c|c|c|c|c|}
\hline$\left(x_{1}, x_{2}\right)$ & $x_{1}=0.2$ & $x_{2}=0.4$ & $x_{3}=0.6$ & $x_{4}=0.8$ \\
\hline \hline$x_{1}=0.2$ & 0.0126166 & 0.0116479 & 0.0106793 & 0.00971059 \\
\hline$x_{2}=0.4$ & 0.00924416 & 0.0108257 & 0.0124072 & - \\
\hline$x_{3}=0.6$ & 0.00587172 & 0.0100035 & - & - \\
\hline$x_{4}=0.8$ & 0.00249928 & - & - & - \\
\hline
\end{tabular}

Table 3. Function $u\left(x_{1}, x_{2}\right)$ with 20 iterations by the Newton method and the step size $h=0.25$.

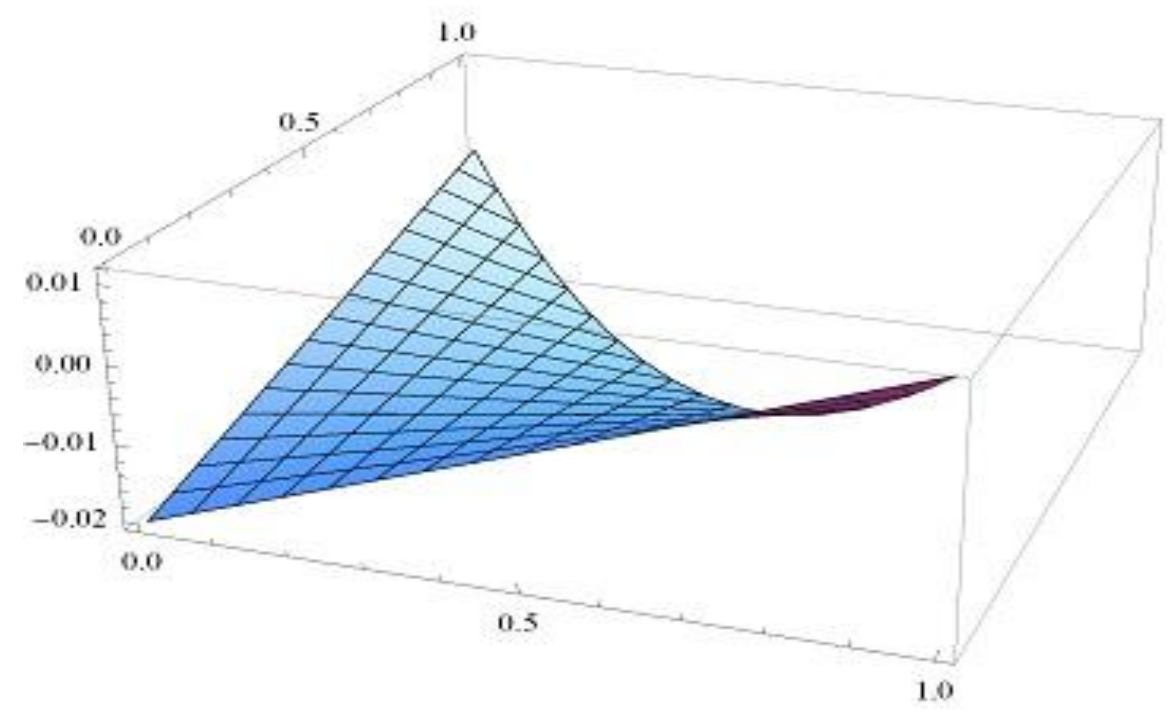

Figure 1. Function $u\left(x_{1}, x_{2}\right)$ in the interval $0,1 \times[0,1-x]$ with the step size $h=0.5$. 


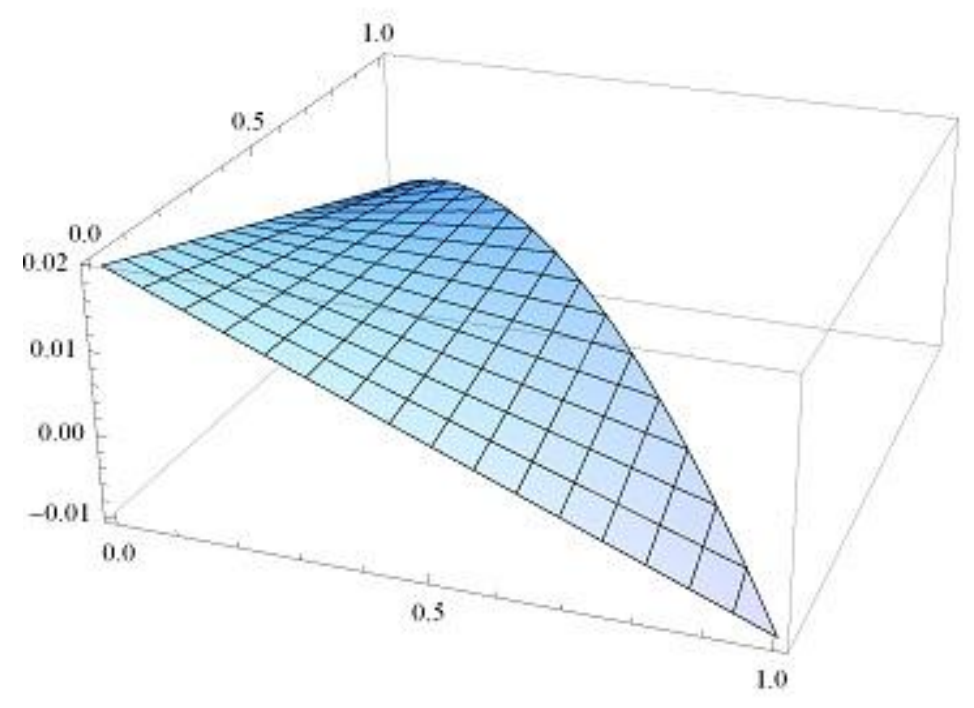

Figure 2. Function $u\left(x_{1}, x_{2}\right)$ in the interval $0,1 \times[0,1-x]$ with the step size $h=0.25$.

Acknowledgements. This work has been supported by a grant from "Islamic Azad university, South Tehran Branch" entitled "Application of numerical integration methods for solving differential equation problems".

\section{References}

\subsection{Journal Articles}

[1] K. Hollig, U. Reif, Nonuniform web-splines, Computer Aided Geometric Design 20(2003)277294.

[2] K. Hollig, U. Reif, J. Wipper, Weighted extended B-spline approximation of Dirichlet problems, SIAM J. Numer. Anal. 39-2(2000) 442-462.

[3] V. V. K. Srinivas Kumar, B. V. Ratish Kumar, P. C. Das, Weighted extended B-spline method for the approximation of stationary Stokes problem, Com. Math. Appl. 186(2006)335-348.

[4] A. Zakeri, M. Asadi Abchouyeh, Solving an elliptic control problem with BEM and FEM, The Journal of Mathematics and Computer Science 3(2012)448-455.

\subsection{Books}

[5] O. Axelsson, V. A. Barker, Finite element solution of boundary value problems, Academic Press, 1984.

[6] M. Hinze, R. Pinnau, M. Ulbrich, S. Ulbrich, Optimization with PDE constraints, ISBN 978-14020-8839-1. 2009.

[7] K. Hollig, Finite element methods with B-splines, SIAM, Philadelphia, 2003.

[8] K. Hollig, U. Reif, J. Wipper, B-spline approximation of Neumann problems, Preprint 2001-2, Universitat Stuttgart.

[9] M. Weiser, T. Ganzler, A. Schtela, A control reduced primal interior point method for PDE constrained optimization. ZIB Report 04-38, Zuse Institute Berlin, 2004. 